\title{
A survey of the sperm whale (Physeter catodon) commensal microbiome
}

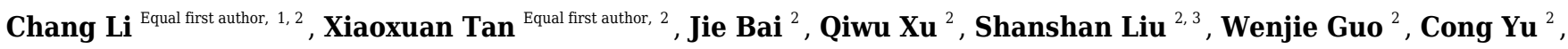 \\ Guangyi Fan $^{2,4,5}$, Yishan Lu ${ }^{6}$, He Zhang ${ }^{2}$, Huanming Yang ${ }^{1,5,7}$, Jianwei Chen ${ }^{\text {Corresp., } 2 \text {, Xin Liu }}{ }^{\text {Corresp. 2, 5, 7, } 8}$ \\ ${ }^{1}$ BGI Education Center, University of Chinese Academy of Sciences, Shenzhen, China \\ 2 BGI-Qingdao, BGI-Shenzhen, Qingdao, China \\ 3 Shandong Technology Innovation Center of Synthetic Biology, Qingdao, China \\ ${ }^{4}$ State Key Laboratory of Quality Research of Chinese Medicine and Institute of Chinese Medical Sciences, University of Macau, Macau, China \\ 5 BGI-Shenzhen, Shenzhen, China \\ 6 Guangdong Ocean University, Shenzhen, China \\ 7 China National GeneBank, BGI-Shenzhen, Shenzhen, China \\ 8 State Key Laboratory of Agricultural Genomics, Shenzhen, China \\ Corresponding Authors: Jianwei Chen, Xin Liu \\ Email address: chenjianwei@genomics.cn, liuxin@genomics.cn
}

Background. Mammalian commensal microbiota play important roles in the health of its host. In comparison to terrestrial mammals, commensal microbiota of marine mammals is mainly focused on the composition and function of skin and gut microbiota, with less attention paid to the health impact of bacteria and viruses. Previous studies on sperm whales (Physeter catodon) have affirmed their important phylogenetic position; however, studies on their commensal microbiota have not been published, due to difficulty in sample collection.

Methods. Here, we sequenced the metagenomes of blood, muscle and fecal samples from a stranded sperm whale using the BGISEQ-500 platform. We compared the diversity and abundance of microbiomes from three different tissues and tried to search pathogenic bacterial and virulence genes probably related to the health of the sperm whale. We also performed 16S rDNA sequencing of the fecal sample to compare to published gut metagenome data from other marine mammals.

Results. Our results demonstrated notable differences in species richness and abundance in the three samples. Extensive bacteria, including Enterococcus faecium, Fusobacterium nucleatum, Pseudomonas aeruginosa, Streptococcus anginosus, Streptococcus pneumoniae, and Streptococcus suis, and five toxigenic Clostridium species usually associated with infection, were found in the three samples. We also found the taxa composition of sperm whale gut microbiota was similar to that of other whales, suggesting co-evolution with its host. This study is the first report of the sperm whale gut microbiome, and provides a foundation for the pathogen detection and health assessment of the sperm whale. 


\section{A survey of the sperm whale (Physeter catodon) 2 commensal microbiome}

3

4 Chang $\mathrm{Li}^{1,2, *}$, Xiaoxuan $\mathrm{Tan}^{2,}{ }^{*}$, Jie Bai ${ }^{2}$, Qiwu $\mathrm{Xu}^{2}$, Shanshan $\mathrm{Liu}^{2,3}$, Wenjie $\mathrm{Guo}^{2}$, Cong $\mathrm{Yu}^{2}$, 5 Guangyi Fan ${ }^{2,4,5}$, Yishan Lu ${ }^{6}$, He Zhang ${ }^{2}$, Huanming Yang ${ }^{1,4,7}$, Jianwei Chen ${ }^{2}$, Xin Liu ${ }^{2,4,7,8}$

6

7

Correspondence authors:

Xin Liu ${ }^{2,4,7,8}$, Jianwei Chen ${ }^{2}$

Hengyunshan Road, Qingdao, Shandong, 266555, China.

Email address: liuxin@genomics.cn, chenjianwei@genomics.cn.

\section{Abstract}

Background. Mammalian commensal microbiota play important roles in the health of its host. In comparison to terrestrial mammals, commensal microbiota of marine mammals is mainly focused on the composition and function of skin and gut microbiota, with less attention paid to the health 
27

28

29

impact of bacteria and viruses. Previous studies on sperm whales (Physeter catodon) have affirmed their important phylogenetic position; however, studies on their commensal microbiota have not been published, due to difficulty in sample collection.

Methods. Here, we sequenced the metagenomes of blood, muscle and fecal samples from a stranded sperm whale using the BGISEQ-500 platform. We compared the diversity and abundance of microbiomes from three different tissues and tried to search pathogenic bacterial and virulence genes probably related to the health of the sperm whale. We also performed $16 \mathrm{~S}$ rDNA sequencing of the fecal sample to compare to published gut metagenome data from other marine mammals.

Results. Our results demonstrated notable differences in species richness and abundance in the three samples. Extensive bacteria, including Enterococcus faecium, Fusobacterium nucleatum, Pseudomonas aeruginosa, Streptococcus anginosus, Streptococcus pneumoniae, and Streptococcus suis, and five toxigenic Clostridium species usually associated with infection, were found in the three samples. We also found the taxa composition of sperm whale gut microbiota was similar to that of other whales, suggesting co-evolution with its host. This study is the first report of the sperm whale gut microbiome, and provides a foundation for the pathogen detection and health assessment of the sperm whale.

\section{Introduction}

Microorganisms exist widely in mammal bodies and the environment, and they are indispensable to the health of mammals (Cho \& Blaser 2012; Human Microbiome Project 2012; McFall-Ngai 2013). Marine mammals are emblematic members of aquatic ecosystems, but marine mammal populations are declining gradually, and may be progressively impacted by climate change, environmental pollution, and direct (e.g. hunting) and indirect (e.g. habitat exploitation) anthropogenic activities (Moore 2008; Pompa 2011). There is growing concern about the current health status and life habitat of marine mammals. The study of marine mammalian symbiotic microbes is important to understand the health of marine mammals and their conservation.

Until now, investigations of marine mammalian symbiotic microbes were mainly focused on 
54 the composition and function of skin microbiota communities in order to examine the potential for 55 a core bacterial community and its variability with specific host or environmental factors (Apprill 56 2014; Hooper 2019; K. C. Bierlich 2018). The composition of the gut microbiomes of marine 57 mammals have also been extensively studied, focusing on the putative functionality of these symbiotic communities, differences between marine mammals, and correlationship between marine mammals' diet and evolution (Bik 2016; Erwin 2017; Merson 2014; Nelson 2013; Sanders 2015). As far as diseases in marine mammals have been concerned, few studies have been conducted on the health impact of bacteria and viruses on marine mammals, which might be limited due to the difficulties in sample collection and the opportunistic nature of stranding events (Godoy-Vitorino 2017; Van Bressem 2014). More marine mammals' samples are required to confirm these studies and apply this knowledge to conservation and rehabilitation.

The sperm whale (Physeter catodon) is the largest toothed whale and the only living member of genus Physeter (Marino 2004). As a representative species for Physeteridae, Odontoceti and Cetacea, they have unique adaptations and a phylogenetically important position (Wesley C. Warren 2018). They feed on several species, most notably the squid, but also octopuses, and fish such as demersal rays (Smith \& Whitehead 2000). Recently, the chromosome level genome sequence of the sperm whale has been published (Fan 2019), providing a good genetic foundation for studying its conservation and population structure. However, the commensal microbiome of sperm whale based on metagenomic sequencing has not been reported yet. The diversity, composition and structure of sperm whale symbiotic microbiota are not known.

Hence, a survey of the sperm whale commensal microbiome is very important for understanding its specific microorganisms and finding some possible pathogenic microorganisms related to its health. In this study, we collected a female stranded sperm whale and characterized the blood, muscle and fecal microbiome using metagenomic sequencing. We also screened for pathogenic microorganisms and virulence genes which might be related to its health in these samples. Additionally, we investigated its gut microbiomes using 16S community sequence analysis to detect the unique microbiome taxa composition by comparing it to published cetacean 
81

82

83

84

85

86

87

88

89

90

91

92

93

94

95

96

97

98

99

100

101

102

103

104

105

106

107

microbiomes. Our reference metagenome data and the potential pathogenic microbes could be used to further monitor and evaluate the health of sperm whales and other marine mammals.

\section{Materials and Methods}

\section{Sample collection and DNA extraction}

The present study sampled a living female sperm whale stranded near the bay area of Huizhou City in southern China on 12 $2^{\text {th }}$ March 2017, and we collected a blood sample of about $5 \mathrm{ml}$ from the vein via syringe (Supplementary Figure S1). The whale died on $15^{\text {th }}$ March, approximately 79 $\mathrm{h}$ after rescue. The dead animal was salvaged and post-mortem analysis was performed according to standard protocols (Kuiken T 1991). We collected fecal and muscle samples during the necropsy. We dissected the intestine with sterile scissors and forceps, then we used a swab to collect about $2 \mathrm{~g}$ of fecal matter from inside the incision to a sterile plastic tube. To collect a muscle sample, we cut the sperm whale cortex tissue and about $5 \mathrm{~g}$ of muscle tissue was collected with scissors. Each sample was immediately stored in $-20^{\circ} \mathrm{C}$ freezers before transport to the laboratory within $12 \mathrm{~h}$ for further investigation.

The blood sample was combined with RNAlater to dissolve eukaryotic cells, and then centrifuged to obtain bacteria cells to extract DNA. Fecal and muscle sample DNA extractions were performed using the QIAamp DNA Stool Mini Kit (Qiagen, Valencia, CA, USA). To eliminate RNA contamination, DNase-free RNase was used to treat extracts. Finally, DNA quantity was determined using a Qubit 3.0 fluorometer, and DNA integrity was evaluated by gel electrophoresis (Pan 2018). All lab work in this study was conducted in a sterile flow hood to prevent contamination. Blood and other samples were dealt in different laboratories to prevent same microbiota contamination. This study was approved by the Institutional Review Board of BGI (NO.BGI-R052-3， NO.FT 17160 and NO.SIDSSE-SYLL-MMMBL-01).

\section{Library construction and sequencing}

We totally constructed three metagenome pair-end libraries for the three samples and one $16 \mathrm{~S}$ 
108

109

110

111

112

113

114

115

116

117

118

119

120

121

122

123

124

125

\section{6}

127

128

129

130

131

132

133

134

rDNA amplicon library for the fecal sample. To construct metagenome sequencing libraries, the extracted DNA was sheared into fragments between $500 \mathrm{bp}$ and $\sim 800 \mathrm{bp}$ in size. Fragments between $150 \mathrm{bp}$ and $250 \mathrm{bp}$ were selected using AMPure XP beads (Agencourt, Beverly, U.S.) and then repaired using T4 DNA polymerase, (ENZYMATICS, Beverly, U.S.) to obtain blunt ends which were then 3-adenlyated to create sticky ends. These DNA fragments were ligated at both ends to T-tailed adapters and amplified for eight cycles. These amplification products were subjected to a single-strand circularization process using T4 DNA Ligase to generate a singlestranded circular DNA library. For constructing 16S V4 rDNA sequencing library, binding sites for sequencing primers of BGISEQ-500 were added at the 5' end of V4 region common primers (515F: GTGCCAGCMGCCGCGGTAA，806R: GGACTACHVGGGTWTCTAAT). The PCR products with target bands were mixed in equal amounts, and 2\% agarose gel was used for electrophoresis and gel cutting, and then were subjected to generate a single-stranded circular DNA library.

All libraries were sequenced on the BGISEQ-500 platform in the paired-end model with 100 bp length reads for metagenome in BGI-Shenzhen and $150 \mathrm{bp}$ for $16 \mathrm{~S}$ rDNA library in BGIQingdao. Different sequencing machines were used for different libraries to prevent same microbiota contamination.

\section{Metagenome assembly and taxonomic assignment.}

Adaptor contamination, low quality reads and duplication for metagenomes were filtered from the raw reads by SOAPnuke (1.5.6) (Chen 2018), and the metagenome remaining reads were removed to eliminate host DNA on the basis of sperm whale reference (Fan 2019) using SOAP2 (Version 2.21) (Gu 2013) with the parameters "-v 8 -m 4". To account for the possibility of contamination with human-associated microorganisms, clean data were mapped to the human genome, and samtools (Version 1.9) with the parameters "view -q 30 -F 4 -F 256" was used to account mapping reads. After removing the host data, the unmapped metagenome data of $16 \mathrm{~Gb}, 53 \mathrm{~Gb}$ and $15 \mathrm{~Gb}$ data for the blood, fecal and muscle samples, respectively, were subjected to further analysis. We 
135

136

137

138

139

140

141

142

143

144

145

146

147

148

149

150

151

152

\section{3}

154

155

156

157

158

159

160

161

then used metaSPAdes (Version 3.10.1) (Nurk 2013) to assemble each sample separately. MetaGeneMark (Version 3.38) (Zhu 2010) was used to identify the coding sequences (CDSs) of the assembled sequences.

Taxonomic assignment of the predicted genes was carried out using BLAST $+($ Version 2.2.26, best match according to BlastN, evalue $<1$ e-5, coverage $>50 \%$ ) by aligning against the prokaryotic representative genomes (Version 201608) and non-redundant nucleotide (NT) database (Version 20170616) (Zhao 2018). The reads were then aligned to the predicted genes using Bowtie2 (Version 2.2.5) (Langmead \& Salzberg 2012), thus the sequence-based gene abundance profiling was calculated using PathoScope (Version 2.0.6) (Francis 2013). The relative abundances of species were calculated from the relative abundance of their respective genes, and the microbes with a relative abundance below 1e-8 were removed to reduce contamination (Guo 2018).

The blood, muscle and fecal clean reads were aligned to the VFDB (Virulence Factors of Bacterial Pathogens) database using Bowtie2 (Version 2.2.5) and the blood assembly contigs were aligned using blat (Version 3.2.1). The Virulence Factors identified in the two results that were annotated to Clostridium novyi and Clostridium botulinum were selected. The core bacterial virulent genes were aligned by MUSCLE (Version 3.8.31) with the same bacteria genome from NCBI and the trees were built by FastTree (Price 2009).

\section{S rDNA community sequence analysis}

In order to compare the gut microbiota with that of other marine mammals, we first collected the 16S V4 rDNA tags of the fecal samples of six species from the NCBI and EMBI database (see more data details in Supplementary Table S1). Then the tags were clustered to the OTUs (Operational Taxonomic Units) using USEARCH (Version 7.0.1090) (Rognes 2016) with a 97\% threshold. Taxonomic assignment of OTU sequences were classified using Ribosomal Database Project (RDP) Classifier (v.2.2) trained on the RDP database (version trainset14_032015) (Cole 2014), using 0.8 confidence values as a cutoff. The data of sperm whale fecal sample 16S rDNA amplicon was discarded to eliminate the low quality and adapter pollution, then paired-end reads 
162

163

164

165

166

167

168

169

170

171

172

173

174

175

176

177

178

179

180

181

182

183

184

185

186

187

188

with overlap were merged to tags using FLASH (v1.2.11). Finally, all tags were mapped into the OTU representative sequences using USEARCH GLOBAL to obtain the OTUs and species abundance profiles. Bray-Curtis distance was used to estimate $\beta$-diversity (between-sample diversity).

\section{Results}

Community diversity and structure of the samples' microbiome.

To obtain a reference metagenomic sequences of the three tissues, we performed a strict data quality control and obtained $\sim 16 \mathrm{~Gb}, \sim 53 \mathrm{~Gb}$ and $\sim 15 \mathrm{~Gb}$ high-quality data of blood, fecal and muscle samples, respectively (Supplementary Table S2). Subsequently, we assembled the metagenomic reads of the three tissues, with an assembly size of $\sim 37 \mathrm{Mb}, \sim 470 \mathrm{Mb}$ and $\sim 151 \mathrm{Mb}$ for blood sample, fecal sample and muscle sample, respectively (Table 1). A total of 1,715 microorganisms were found in the three tissues, among which the microbial species in the fecal samples were the most abundant. There were notable differences in species richness and abundance in the different samples (Figure 1A).

In all three tissues, only 113 microbial species were detectable, and they were those belonging to seven phyla Firmicutes, Bacteroidetes, Deferribacteres, Spirochaetes, Fusobacteria, Proteobacteria and Tenericutes (Supplementary Table S3). A total of 21 phyla and 74 genera were identified in at least two tissues (Figure 1B). Each sample had a dominance of different taxa; for instance, the blood sample was dominated by Firmicutes. In comparison with blood and muscle samples, the fecal sample was more abundant in Bacteroidetes and Firmicutes. The muscle sample shared fewer genera when compared to the other two samples.

\section{Detection of potentially pathogenic microorganisms}

To detect potentially pathogenic microbes of the sperm whale, we screened the pathogenic bacterial species in the metagenome sequence data of three tissues. We found pathogenic microbes including Enterococcus faecium, Fusobacterium nucleatum, Pseudomonas aeruginosa, 
189

190

191

192

193

194

195

196

197

198

199

200

201

202

203

204

205

206

207

208

209

210

211

212

213

214

215

Streptococcus anginosus, Streptococcus pneumoniae, Streptococcus suis, and five toxigenic Clostridium in the bloodstream and intestine, and six of them appeared in muscle tissue (Table 2) (Chevalier 2017; Engholm 2017; Fortier 2017; Gao 2018; Han 2015; Okwumabua 2017). Then we aligned the blood, muscle and fecal assembly results and sequencing data against the VFDB databases to identify bacterial virulence genes (Chen LH 2016). Twelve virulence genes were found in Clostridium novyi and Clostridium botulinum. By aligning the pathogenic genes to all strain sequences of $C$. novyi and $C$. botulinum, we found the nearest bacteria to them were $C$. novyi A str. 4540 (GCA_000724445) and C. botulinum C str. Stockholm (GCA_000219255) from Sweden (Figure 2A and 2B).

\section{Comparison of gut microbiome in different marine mammals}

The composition of mammalian gut microbiota could be shaped by host diet, age and phylogenetic position (Hullar \& Fu 2014; Muegge 2011). To profile the unique gut microbiota of sperm whales, we compared the composition of gut microbiome between sperm whales and other marine mammals. A total of 122 bacterial Operational Taxonomic Units (OTUs) were identified in the sperm whale's gut microbiota (Supplementary Table S4). The majority of microbes were members of the phyla Bacteroidetes (51.49\% of the total) and Firmicutes (31.18\% of the total), as well as the phyla Euryarchaeota, Spirochaetes and Verrucomicrobia (Figure 3A). The sperm whale's sample had either very few or no reads assigned to Proteobacteria, which was comparatively common among six other marine mammals.

We also calculated these compositional dissimilarities using $\beta$-diversity (Whittaker 1960), and built cluster trees using $\beta$-diversity to disentangle the effect of different factors shaping community assembly at different phylogenetic scales. The Bray-Curtis distance in mammalian gut microbiomes showed that the sperm whale was closest to the Beluga Whale (Delphinapterus leucas) and furthest from the manatee (Florida manatee), which is consistent with the biological evolutionary tree of these marine species (Figure 3B) (Groussin 2017). 
216 Discussion

217 Here, the first microbiome inventory of a stranded sperm whale was presented. Among previous

218

219

220

221

222

223

224

225

226

227

228

229

230

231

232

233

234

235

236

237

238

239

240

241

242 metagenomic studies, few have focused on the commensal microbiome of stranded marine mammals, possibly due to the high contamination risk during sample collection (Godoy-Vitorino 2017). In this study, we significantly focused on sperm whale salvage in order to ensure that in the collection of fecal and muscle samples, there was no contamination by the external environment. To monitor DNA extraction kits and other laboratory reagent contamination (Lusk 2014; Susannah J Salter 2014), we set up a negative control for the meta 16S rDNA library (Supplementary Figure S2) and removed microbes with relative abundance below 1e-8 for metagenomic data (Guo 2018). To reduce possibility contamination with human-associated microorganisms, we removed the reads which can map to human genome with high quality (Hooper 2019). The commensal microbiome of the stranded sperm whale in this study contained five main phyla ( $>1 \%$ relative abundance), which were also reported in pygmy (Kogia breviceps) and dwarf (K. sima) sperm whales, corroborating the validity of contamination control in this study (Erwin 2017; GodoyVitorino 2017).

The pathogens detected in this study, including E. faecium, $P$. aeruginosa, $S$. anginosus, $S$. pneumoniae, S. suis, and F. nucleatum, are opportunistic pathogens and can cause blood infection and immunity damage (Asam \& Spellerberg 2014; Engholm 2017; Gao 2018; Mulcahy 2014; Prithiviraj 2005). F. nucleatum and five toxigenic Clostridium were found in all three samples, and F. nucleatum is associated with a wide spectrum of human diseases (Bashir 2016; Han 2015; Signat 2011). Twelve virulence genes were found in C. novyi and C. botulinum which were the nearest two highly virulent bacteria of C. novyi A str. 4540 (GCA_000724445) and C. botulinum C str. Stockholm (GCA_000219255) from Sweden (Figure 2A and 2B). C. novyi A can cause Black disease and is characterized as lethal and necrotizing (Kahn 2005; Skarin \& Segerman 2014), and C. botulinum $C$ (group III) can cause diseases in animals. This suggests that $C$. novyi and $C$. botulinum found in this study may also affect host health (Skarin H 2011).

Many pathogenic bacteria have been found in humans and other economic animals (Baumler 
243 \& Sperandio 2016), although the reports of them in marine mammals were limited. Live stranding 244 causes a number of Cetacean deaths (CM Kemper 2005; Karen Evans 2002), and bacterial 245 infection has been suggested to be an important and underestimated factor leading to stranding of 246 marine mammals (Cools 2013; Cowan 2001). The pathogens we detected also can be used to 247 monitor the health of marine mammals. Additionally, we found the pathogenic nematodes 248 Elaeophora elaphi in the metagenome data of blood that was supported by our dissection result 249 (Table 2, Supplementary Figure S3) (Hernandez Rodriguez 1986). This also proved that this is a comprehensive method to understand the overall health of the marine mammals.

Additionally, the order of pathogenic species found in fecal samples were also identified in meta 16S rDNA data analysis results. Genomic DNA was extracted from the blood sample and other samples from different laboratories, and metagenomic library construction and meta $16 \mathrm{~S}$ library construction were also independent. Thus, the pathogenic microorganisms in the three samples were real components of the sperm whale commercial microbiome (Hooper 2019).

Moreover, when comparing the gut microbes of other marine mammals, we performed 16S V4 sequencing for the sperm whale fecal sample instead of using metagenomics data. Since most of the published gut microbe data of marine mammals are 16S data, we maintained consistency with them to ensure the accuracy of the analysis results (Sanders 2015). High abundance of Bacteroidetes and Firmicutes identified in the gut of the sperm whale were similar with pygmy and dwarf sperm whales, indicating similar richness and evenness of gut microbiomes in these closely related species (Erwin 2017; LANGER 2001; Sanders 2015; Zhu 2011). Interestingly, the $\beta$-diversity phylogenetic tree is consistent with the evolution of host, suggesting that sperm whales and their gut microbiota have a coevolutionary relationship that was also consistent with reports from previous research (Groussin 2017; Sanders. 2015). In the future, more gut microbiome data of sperm whales and other marine mammals should be studied to identify the structural determinants of gut microbiome composition in marine mammals.

\section{Conclusions}


270

271

272

273

274

275

276

277

278

279

280

281

282

283

284

285

286

287

288

289

290

291

292

293

294

295

296

297

298

299

300

301

302

As the first metagenomic survey of sperm whale blood, fecal and muscle microbiome using nextgeneration sequencing, our results showed the differences in symbiotic microorganisms among the three tissues, and demonstrated the gut microbiota of sperm whales has a coevolutionary relationship with its host. The pathogens we detected in the blood, muscle and fecal samples may cause some health problems in the sperm whale, knowledge which will contribute to the monitoring of the health of marine mammals.

\section{Acknowledgements}

We thank Guangdong Ocean University and the Institute of Deep-sea Science and Engineering Chinese Academy of Sciences for collecting samples. We thank Xiangqun Chi and Santasree Banerjee from BGI-Qingdao for revising the manuscript. We thank Shandong Technology Innovation Center of Synthetic Biology for their advises to support this work.

\section{Reference}

Apprill A, Robbins J, Eren AM. 2014. Humpback whale populations share a core skin bacterial community: towards a health index for marine mammals? PLoS One 9:e90785. 10.1371/journal.pone.0090785

Asam D, and Spellerberg B. 2014. Molecular pathogenicity of Streptococcus anginosus. Mol Oral Microbiol 29:145155. 10.1111/omi.12056

Bashir A, Miskeen AY, Hazari YM. 2016. Fusobacterium nucleatum, inflammation, and immunity: the fire within human gut. Tumour Biol 37:2805-2810. 10.1007/s13277-015-4724-0

Baumler AJ, and Sperandio V. 2016. Interactions between the microbiota and pathogenic bacteria in the gut. Nature 535:85-93. 10.1038/nature18849

Bik EM, Costello EK, Switzer AD. 2016. Marine mammals harbor unique microbiotas shaped by and yet distinct from the sea. Nat Commun 7:10516. 10.1038/ncomms10516

Chen LH ZD, Liu B, Yang J and Jin Q. 2016. VFDB 2016 hierarchical and refined dataset for big data analysis-10 years. Nucleic Acids Res 44(Database issue):D694-D697.

Chen Y, Chen Y, Shi C. 2018. SOAPnuke: a MapReduce acceleration-supported software for integrated quality control and preprocessing of high-throughput sequencing data. Gigascience 7:1-6. 10.1093/gigascience/gix120

Chevalier S, Bouffartigues E, Bodilis J. 2017. Structure, function and regulation of Pseudomonas aeruginosa porins. FEMS Microbiol Rev 41:698-722. 10.1093/femsre/fux020

Cho I, and Blaser MJ. 2012. The human microbiome: at the interface of health and disease. Nat Rev Genet 13:260270. $10.1038 / \mathrm{nrg} 3182$

CM Kemper AF, SE Gibbs, M Hill , M Long. 2005. Cetacean captures, strandings and mortalities in South Australia 
1881-2000, with special reference to human interactions. Australian Mammalogy 27(1) 37 - 47.

Cole JR, Wang Q, Fish JA, Chai B. 2014. Ribosomal Database Project: data and tools for high throughput rRNA analysis. Nucleic Acids Res 42:D633-642. 10.1093/nar/gkt1244

Cools P, Haelters J, Lopes dos Santos Santiago G. 2013. Edwardsiella tarda sepsis in a live-stranded sperm whale (Physeter macrocephalus). Vet Microbiol 166:311-315. 10.1016/j.vetmic.2013.05.020

Cowan DF, House, C., House, J.A. 2001. Handbook of Marine Mammal Medicine. Public health:767-778.

Engholm DH, Kilian M, Goodsell DS. 2017. A visual review of the human pathogen Streptococcus pneumoniae. FEMS Microbiol Rev 41:854-879. 10.1093/femsre/fux037

Erwin PM, Rhodes RG, Kiser KB, Keenan-Bateman TF. 2017. High diversity and unique composition of gut microbiomes in pygmy (Kogia breviceps) and dwarf (K. sima) sperm whales. Sci Rep 7:7205. 10.1038/s41598-017-07425-z

Fan G, Zhang Y, Liu X, Wang J, Sun Z, Sun S. 2019. The first chromosome-level genome for a marine mammal as a resource to study ecology and evolution. Mol Ecol Resour. 10.1111/1755-0998.13003

Fortier LC. 2017. The Contribution of Bacteriophages to the Biology and Virulence of Pathogenic Clostridia. Adv Appl Microbiol 101:169-200. 10.1016/bs.aambs.2017.05.002

Francis OE, Bendall M, Manimaran S, Hong C. 2013. Pathoscope: species identification and strain attribution with unassembled sequencing data. Genome Res 23:1721-1729. 10.1101/gr.150151.112

Gao W, Howden BP, and Stinear TP. 2018. Evolution of virulence in Enterococcus faecium, a hospital-adapted opportunistic pathogen. Curr Opin Microbiol 41:76-82. 10.1016/j.mib.2017.11.030

Godoy-Vitorino F, Rodriguez-Hilario A, Alves AL. 2017. The microbiome of a striped dolphin (Stenella coeruleoalba) stranded in Portugal. Res Microbiol 168:85-93. 10.1016/j.resmic.2016.08.004

Groussin M, Mazel F, Sanders JG, Smillie CS. 2017. Unraveling the processes shaping mammalian gut microbiomes over evolutionary time. Nat Commun 8:14319. 10.1038/ncomms14319

Gu S, Fang L, and Xu X. 2013. Using SOAPaligner for Short Reads Alignment. Curr Protoc Bioinformatics 44:11 11 1117. 10.1002/0471250953.bi1111s44

Guo M, Chen J, Li Q, Fu Y, Fan G. 2018. Dynamics of Gut Microbiome in Giant Panda Cubs Reveal Transitional Microbes and Pathways in Early Life. Front Microbiol 9:3138. 10.3389/fmicb.2018.03138

Han YW. 2015. Fusobacterium nucleatum: a commensal-turned pathogen. Curr Opin Microbiol 23:141-147. 10.1016/j.mib.2014.11.013

Hernandez Rodriguez S, Martinez Gomez F. 1986. Elaeophora elaphi n. sp. (Filarioidea: Onchocercidae) parasite of the red deer (Cervus elaphus). With a key of species of the genus Elaeophora. Ann Parasitol Hum Comp 61:457-463. 10.1051/parasite/1986614457

Hooper R, Brealey JC. 2019. Host-derived population genomics data provides insights into bacterial and diatom composition of the killer whale skin. Mol Ecol 28:484-502. 10.1111/mec.14860

Hullar MA, and Fu BC. 2014. Diet, the gut microbiome, and epigenetics. Cancer J 20:170-175. 10.1097/PPO.0000000000000053

Human Microbiome Project C. 2012. Structure, function and diversity of the healthy human microbiome. Nature 486:207-214. 10.1038/nature11234

K. C. Bierlich CM. 2018. Temporal and regional variability in the skin microbiome of humpback whales along the Western Antarctic peninsula. ENVIRONMENTAL MICROBIOLOGY 84:e02574-02517.

Kahn C. 2005. Infectious Necrotic Hepatitis (Black disease). The Merck Veterinary Manual (9th ed) Whitehouse 
Station, New Jersey: Merck \& Co ISBN 978-0-911910-50-6. OCLC 57355058.

Karen Evans MM, Mark Hinde. 2002. Three mass strandings of sperm whales (physeter macrocephalus) in southern Australian waters. MARINE MAMMAL SCIENCE 18(3):622-643.

Kuiken T HM. 1991. Proceedings of the First ECS Workshop on Cetacean Pathology : dissection techniques and tissue sampling. Leiden Netherlands: [European Cetacean Society]; .

LANGER P. 2001. Evidence from the digestive tract on phylogenetic relationships in ungulates and whales. Institut fuÈr Anatomie \& Zellbiologie 39 (2001) 77-90.

Langmead B, and Salzberg SL. 2012. Fast gapped-read alignment with Bowtie 2. Nat Methods 9:357-359. 10.1038/nmeth.1923

Lusk RW. 2014. Diverse and widespread contamination evident in the unmapped depths of high throughput sequencing data. PLoS One 9:e110808. 10.1371/journal.pone.0110808

Marino L. 2004. Cetacean brain evolution: multiplication generates complexity. International Journal of Comparative Psychology Vol 17(1), 2004, 1-16.

McFall-Ngai M, Hadfield MG, Bosch TC. 2013. Animals in a bacterial world, a new imperative for the life sciences. Proc Natl Acad Sci U S A 110:3229-3236. 10.1073/pnas.1218525110

Meehan CJ, Langille MG, and Beiko RG. 2015. Frailty and the Microbiome. Interdiscip Top Gerontol Geriatr 41:54-65. 10.1159/000381162

Merson SD, Ouwerkerk D, Gulino LM. 2014. Variation in the hindgut microbial communities of the Florida manatee, Trichechus manatus latirostris over winter in Crystal River, Florida. FEMS Microbiol Ecol 87:601-615. 10.1111/1574-6941.12248

Moore SE. 2008. Marine mammals as ecosystem sentinels. Journal of Mammalogy 89:534-540. 10.1644/07-mamms-312r1.1

Muegge BD, Kuczynski J, Knights D. 2011. Diet drives convergence in gut microbiome functions across mammalian phylogeny and within humans. Science 332:970-974. 10.1126/science.1198719

Mulcahy LR, Isabella VM, and Lewis K. 2014. Pseudomonas aeruginosa biofilms in disease. Microb Ecol 68:1-12. 10.1007/s00248-013-0297-x

Nelson TM, Rogers TL, Carlini AR, and Brown MV. 2013. Diet and phylogeny shape the gut microbiota of Antarctic seals: a comparison of wild and captive animals. Environ Microbiol 15:1132-1145. 10.1111/14622920.12022

Nurk S, Bankevich A, Antipov D. 2013. Assembling single-cell genomes and mini-metagenomes from chimeric MDA products. J Comput Biol 20:714-737. 10.1089/cmb.2013.0084

Okwumabua O, Peterson H, Hsu HM, Bochsler P, and Behr M. 2017. Isolation and partial characterization of Streptococcus suis from clinical cases in cattle. J Vet Diagn Invest 29:160-168. 10.1177/1040638717690014

Pan H, Guo R, Zhu J, Wang Q, Ju Y, Xie Y. 2018. A gene catalogue of the Sprague-Dawley rat gut metagenome. Gigascience 7. 10.1093/gigascience/giy055

Pompa S, Ehrlich PR, and Ceballos G. 2011. Global distribution and conservation of marine mammals. Proc Nat/ Acad Sci U S A 108:13600-13605. 10.1073/pnas.1101525108

Price MN, Dehal PS, and Arkin AP. 2009. FastTree: computing large minimum evolution trees with profiles instead of a distance matrix. Mol Biol Evol 26:1641-1650. 10.1093/molbev/msp077

Prithiviraj B, Bais HP, Weir T. 2005. Down regulation of virulence factors of Pseudomonas aeruginosa by salicylic acid attenuates its virulence on Arabidopsis thaliana and Caenorhabditis elegans. Infect Immun 73:5319-5328. 
10.1128/IAI.73.9.5319-5328.2005

Rognes T, Flouri T, Nichols B, Quince C, and Mahe F. 2016. VSEARCH: a versatile open source tool for metagenomics. PeerJ 4:e2584. 10.7717/peerj.2584

Sanders JG, Beichman AC, Roman J, Scott JJ. 2015. Baleen whales host a unique gut microbiome with similarities to both carnivores and herbivores. Nat Commun 6:8285. 10.1038/ncomms9285

Signat B, Roques, C., Poulet, P., Duffaut, D. 2011. Role of Fusobacterium nucleatum in periodontal health and disease. Curr Issues Mol Biol 13, 25-36.

Skarin H HT, Westerberg J, Segerman B. 2011. Clostridium botulinum group III a group with dual identity shaped by plasmids phages and mobile elements. BMC Genomics 12:185.

Skarin H, and Segerman B. 2014. Plasmidome interchange between Clostridium botulinum, Clostridium novyi and Clostridium haemolyticum converts strains of independent lineages into distinctly different pathogens. PLoS One 9:e107777. 10.1371/journal.pone.0107777

Smith SC, and Whitehead H. 2000. The diet of galapagos sperm whales physeter macrocephalus as indicated by fecal sample analysis MARINE MAMMAL SCIENCE 16(2):3 15-325.

Susannah J Salter MJC, Elena M Turek, Szymon T Calus. 2014. Reagent and laboratory contamination can critically impact sequence-based microbiome analyses. BMC Biology 12:87.

Van Bressem MF, Duignan PJ, Banyard A, Barbieri M. 2014. Cetacean morbillivirus: current knowledge and future directions. Viruses 6:5145-5181. 10.3390/v6125145

Wesley C. Warren LK, Alana Alexander. 2018. The Novel Evolution of the Sperm Whale Genome. Genome Biol Evol 10:1063. 10.1093/gbe/evy069

Whittaker RH. 1960. Vegetation of the Siskiyou Mountains, Oregon and California. Ecol Monogr 30, $279-338$.

Zhao L, Huang Y, Lu L, Yang W, Huang T. 2018. Saturated long-chain fatty acid-producing bacteria contribute to enhanced colonic motility in rats. Microbiome 6:107. 10.1186/s40168-018-0492-6

Zhu L, Wu Q, Dai J, Zhang S, and Wei F. 2011. Evidence of cellulose metabolism by the giant panda gut microbiome. Proc Natl Acad Sci U S A 108:17714-17719. 10.1073/pnas.1017956108

Zhu W, Lomsadze A, and Borodovsky M. 2010. Ab initio gene identification in metagenomic sequences. Nucleic Acids Res 38:e132. 10.1093/nar/gkq275

Figure 1. Host-associated microbial communities of sperm whale. (A) A PCA of species with sperm whale blood, fecal and muscle samples. (B)The taxonomic distribution of microbes detected at least in two tissues by metagenomics analysis of sperm whale blood, fecal and muscle samples. The relative abundance genera from the top 20 abundant genera are shown.

Figure 2. Phylogeny tree of all strain sequences virulence genes found in $C$. botulinum and $C$. novyi. (A) Phylogeny tree of four virulence genes found in C. botulinum. All C. botulinum strains genome from NCBI were shown. (B) Phylogeny tree of nine virulence genes found in C. novyi. 
421 All C. novyi strains genome from NCBI were shown.

422

423 Figure 3. Gut microbiomes of sperm whale fecal sample. (A) Phylum composition distribution 424 of sperm whale and six marine mammals' gut microbiomes. Beluga whale (Delphinapterus leucas) 425 and dolphin (Tursiops truncatus) are tooth whale. Humpback whale (Megaptera novaeangliae), 426 sei whale (Balaenoptera borealis), and right whale (Eubalaena glacialis) are baleen whale. 427 Manatee (Florida manatee) is belongs to Sirenia. (B) Phylogenetic tree basic on Bray-Curtis 428 distance in mammalian gut microbiomes.

429

430 
Figure 1 (on next page)

Host-associated microbial communities of sperm whale.

(A) A PCA of species with sperm whale blood, fecal and muscle samples. (B)The taxonomic distribution of microbes detected at least in two tissues by metagenomics analysis of sperm whale blood, fecal and muscle samples. The relative abundance genera from the top 20 abundant genera are shown. 
A

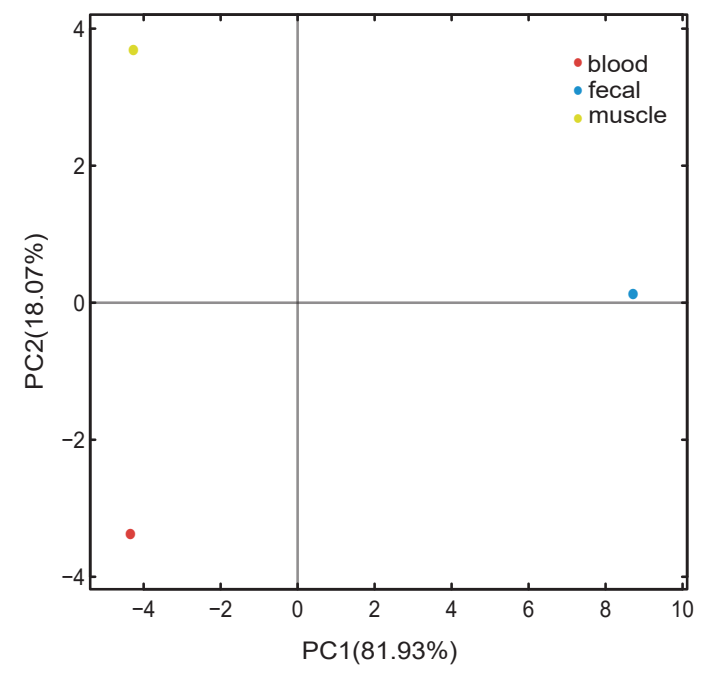

B

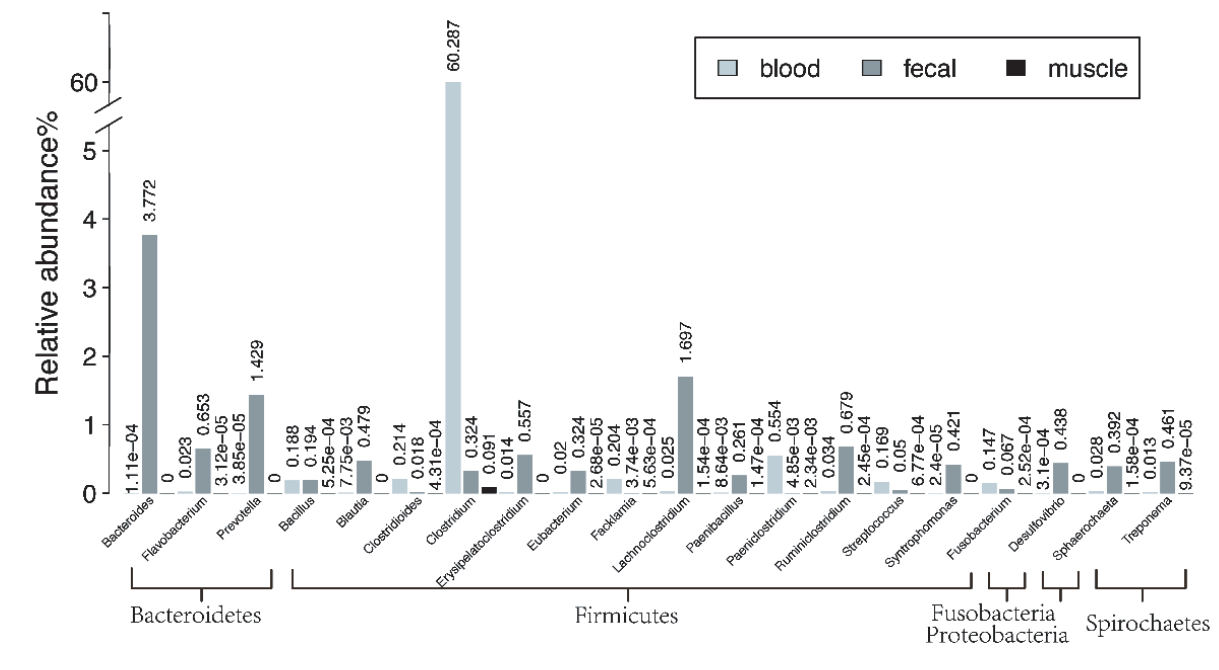


Figure 2 (on next page)

Phylogeny tree of all strain sequences virulence genes found in C. botulinum and $C$. novyi.

(A) Phylogeny tree of four virulence genes found in C. botulinum. All C. botulinum strains genome from NCBI were shown. (B) Phylogeny tree of nine virulence genes found in $C$. novyi. All C. novyi strains genome from NCBI were shown. 
A

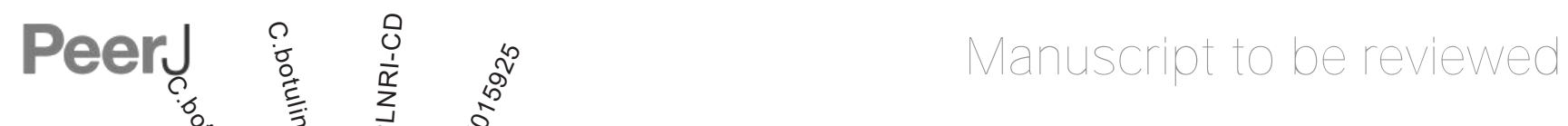

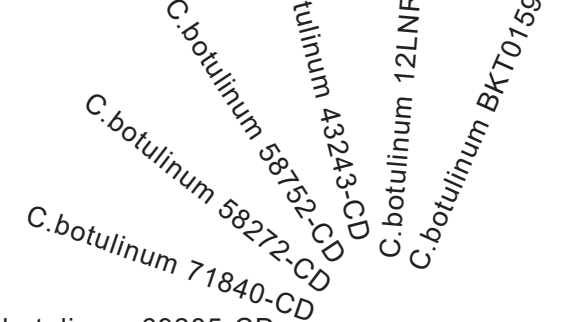

C.botulinum 69285-CD

C. botulinum $50867-C D$

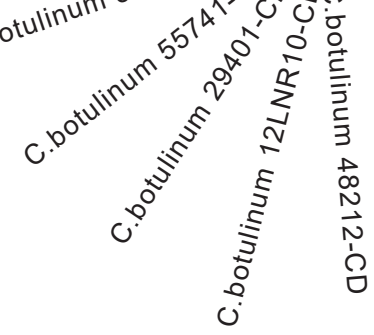

B
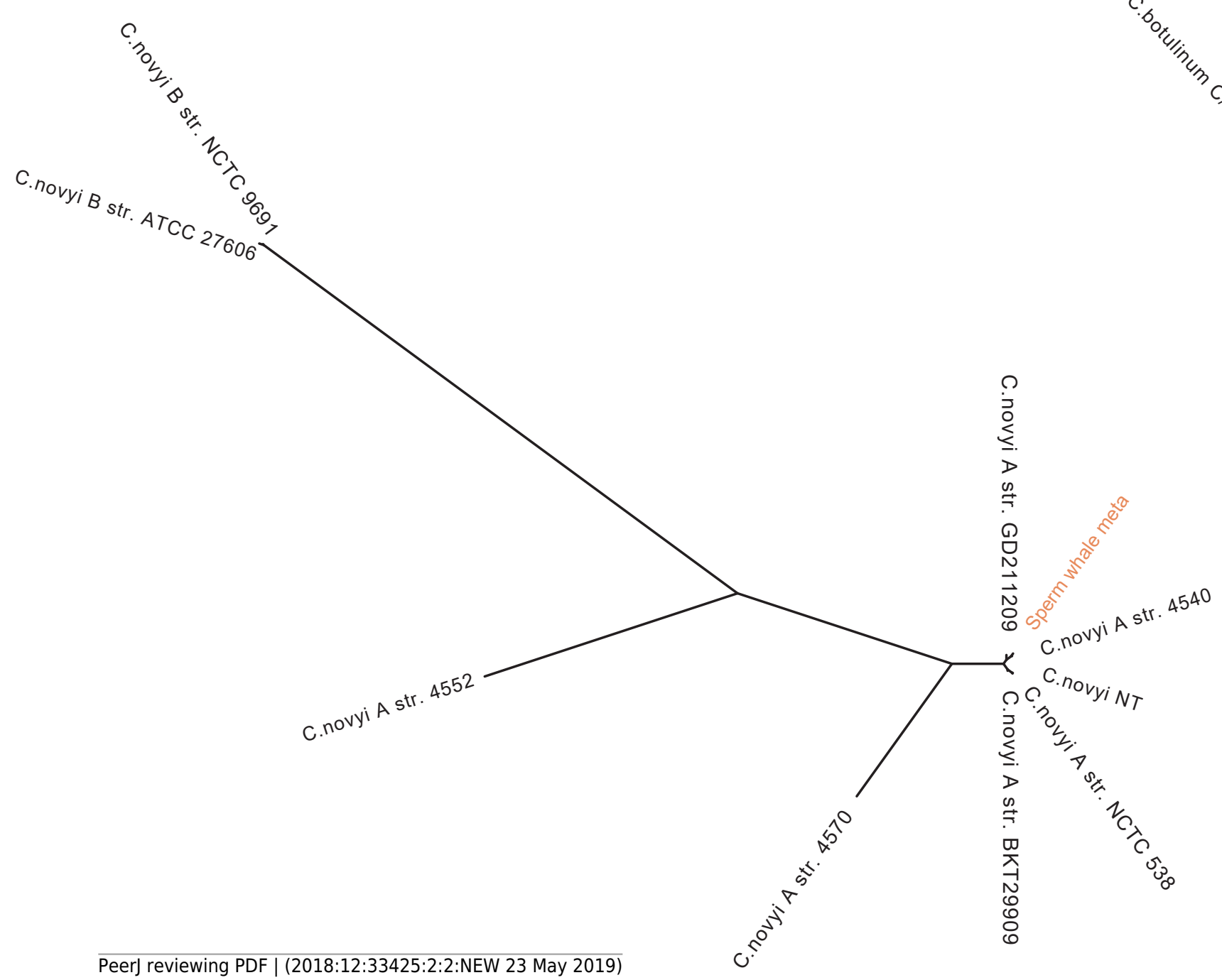
Figure 3 (on next page)

Gut microbiomes of sperm whale fecal sample.

(A) Phylum composition distribution of sperm whale and six marine mammals' gut microbiomes. Beluga whale (Delphinapterus leucas) and dolphin (Tursiops truncatus) are tooth whale. Humpback whale (Megaptera novaeangliae), sei whale (Balaenoptera borealis), and right whale (Eubalaena glacialis) are baleen whale. Manatee (Florida manatee) is belongs to Sirenia. (B) Phylogenetic tree basic on Bray-Curtis distance in mammalian gut microbiomes. 


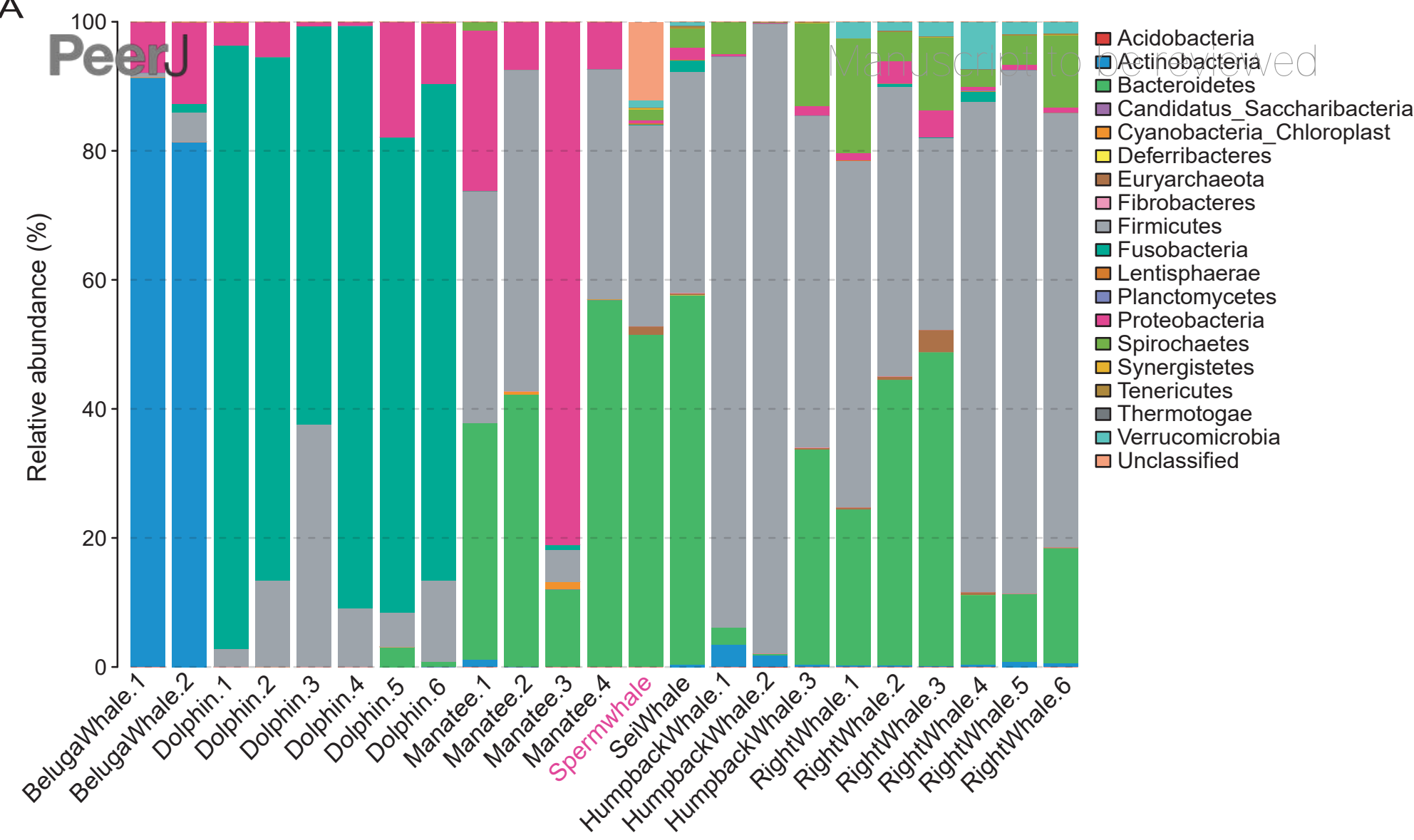

B
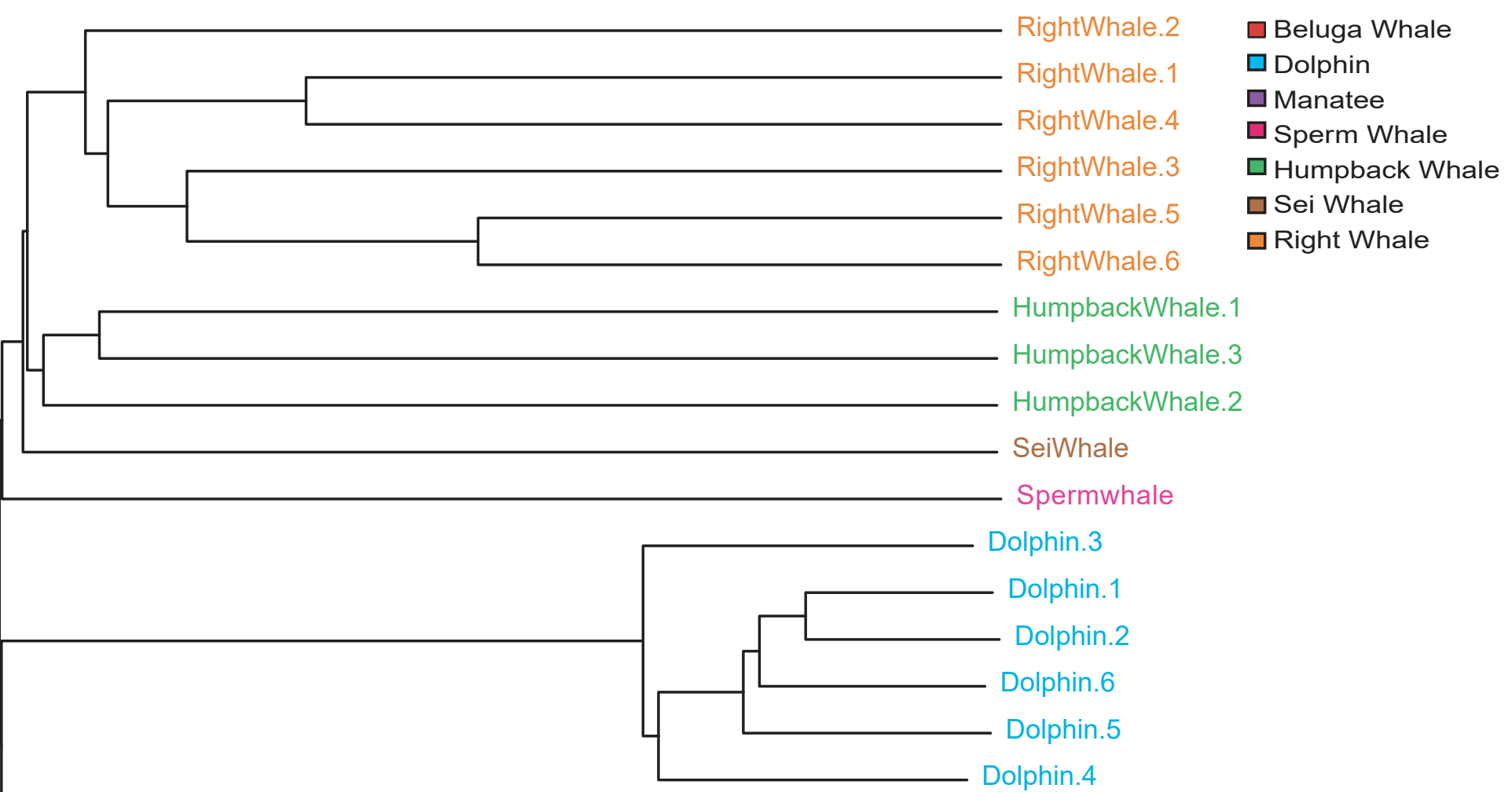

BelugaWhale.1

BelugaWhale.2

Manatee. 3

Manatee.2 


\section{Table $\mathbf{1}$ (on next page)}

Statistics of metagenomics assembly for three tissues. 
Table 1. Statistics of metagenomics assembly for three tissues.

\begin{tabular}{lllllll}
\hline \multirow{2}{*}{ Statistics } & \multicolumn{2}{c}{ Blood } & \multicolumn{2}{c}{ Fecal } & \multicolumn{2}{c}{ Muscle } \\
\cline { 2 - 7 } & Contig & CDS & Contig & CDS & Contig & CDS \\
\hline Total number & 40,086 & 61,992 & 287,646 & 628,309 & 155,131 & 174,718 \\
Total length(Mb) & 37.16 & 27.83 & 470.47 & 375.63 & 151.50 & 43.71 \\
N50 length (bp) & 1,930 & 717 & 5,153 & 963 & 2,060 & 306 \\
N90 length (bp) & 309 & 213 & 498 & 294 & 314 & 123 \\
Max length (bp) & 488,134 & 10,443 & 546,844 & 29,826 & 112,716 & 147,900 \\
Min length (bp) & 200 & 57 & 300 & 57 & 200 & 57 \\
GC content & $32.73 \%$ & $32.94 \%$ & $48.30 \%$ & $49.18 \%$ & $49.97 \%$ & $52.26 \%$ \\
Mapped reads & $91.43 \%$ & $66.33 \%$ & $88.87 \%$ & $57.86 \%$ & $18.57 \%$ & $7.11 \%$ \\
\hline
\end{tabular}

2 
Table 2 (on next page)

Detection of pathogenic bacteria and nematodes in fecal, blood and muscle samples. 
1 Table 2. Detection of pathogenic bacteria and nematodes in fecal, blood and muscle samples.

\begin{tabular}{lccc}
\hline Species & Blood & Fecal & Muscle \\
\hline Clostridium baratii & + & + & + \\
Clostridium botulinum & + & + & + \\
Clostridium novyi & + & + & + \\
Clostridium perfringens & + & + & + \\
Clostridium tetani & + & + & + \\
Enterococcus faecium & + & + & - \\
Fusobacterium nucleatum & + & + & - \\
Pseudomonas aeruginosa & + & + & - \\
Streptococcus anginosus & + & + & - \\
Streptococcus pneumoniae & + & + & - \\
Streptococcus suis & + & + & - \\
Bacteroides fragilis & - & + & - \\
Enterococcus faecalis & - & \multicolumn{2}{c}{} \\
\hline
\end{tabular}

2 